\title{
Reach Out and Touch Someone
}

Wendy A. Kellogg, Queue Advisory Board Member

$\mathbf{T}$ The two killer apps that have best defined the Internet Age have been e-mail and the Web. Now we have a third: IM (instant messaging). Note that two of the three are entirely focused on person-to-person communication-no great surprise since work demands that we find increasingly efficient and effective means to communicate with colleagues, customers, and suppliers. And then out in that big world beyond day-to-day commerce are all those other people we really want to be able to connect directly with in some convenient yet meaningful way. For millions of computer and wireless phone users, IM has become a preferred means for doing just that.

Yet, as it stands, IM is still only in an early developmental stage. Much remains to be done to allow transparent interaction across protocols that still aren't even on speaking terms, provide for more sophisticated filtering and security measures, and enhance the experience of users through new capabilities designed to enable easier collaboration and the use of rich media.

To shed some light on all that, we asked two people who've figured large in the development of both e-mail and IM, respectively, to discuss how those two worlds are apt to influence one another as both continue to evolve. Eric Allman, the CTO at Sendmail, who also just happened to author Sendmail, the world's first Internet Mail program, delivers the questions. Peter Ford, chief architect for Microsoft's MSN Messenger, responds. As both Allman and Ford quickly discovered, there's much in each world to inform the other-with the experience of e-mail suggesting ways for IM developers to create gateways for easy communication across varied protocols, and IM likely to ultimately offer lessons to e-mail developers on how to support rich media and implement better mechanisms for authentication and filtering.

Much work remains to be done to bring together the different IM communities served by the SIP/SIMPLE and XMPP protocols. Joe Hildebrand, the CTO at Jabber, ruminates on exactly what needs to happen for everyone to get along in an article aptly titled "Nine IM Accounts and Counting." Marshall T. Rose, a cofounder of Invisible Worlds and the former CTO of MessageMedia, adds a few thoughts of his own in the Opinion slot.

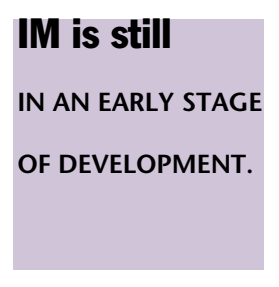

The types of awareness features required for IM to play a more valuable role in office collaboration are the concern that John C.

Tang and James "Bo" Begole from Sun Labs take up in "Beyond Instant Messaging." Frank Jania of IBM Research steps out even further in "Broadcast Messaging: Messaging to the Masses," considering the potential of broadcast messaging to spread the reach of IM to people who don't already know each other-and how this might contribute to community building in an enterprise setting. Meanwhile, Ellen Isaacs chimes in with findings from a large study of monitored IM interactions at AT\&T Labs.

But perhaps you too aren't all that inclined to buy into the IM dream. In that case, you'll be delighted to learn that neither does Jakob Nielsen, the former Sun Microsystems distinguished engineer who equates IM to "one more toxic spill" of information pollution. To find out what other spicy things Nielsen has to say about IM, look for our Curmudgeon space at the back of the magazine.

In addition, this issue contains two articles not part of our special report. "Sentient Data Access via a Diverse Society of Devices," by William Buxton of Buxton Design and colleagues, explores the challenges in letting users take their desktops with them. "Uprooting Software Defects at the Source," by Seth Hallem, David Park, and Dawson Engler of Coverity, digs into the always popular topic of rooting out bugs. $Q$

WENDY A. KELLOGG is manager of social computing (www.research.ibm.com/SocialComputing/) at IBM's T. J. Watson Research Center. Her current work involves defining and promoting the emerging field of social computing, as well as designing, building, and evaluating computer-mediated communication (CMC) systems. She holds a Ph.D. in cognitive psychology from the University of Oregon and is author of numerous papers in the fields of $\mathrm{HCl}$ and CSCW (Computer-Supported Cooperative Work). Kellogg is an ACM Fellow and a member of the National Research Council's Computer Science and Telecommunications Board. 